\title{
Suprapubic granuloma after retropubic sling
}

\begin{abstract}
Background: Stress urinary incontinence is a debilitating condition which occurs in $10-20 \%$ of females. Surgical management of stress urinary incontinence is increasingly common. Suburethral slings are most efficacious in treatment of stress urinary incontinence. Graft materials in these procedures can be synthetic or biologic. Common reported complications include bladder perforation, hematoma, vaginal extrusion of implant, de novo urgency symptoms, urinary tract infections and voiding dysfunction.

Case: A 43-year-old para 5 woman presented to our department with complaint of a left suprapubic growing mass. Her medical history was significant for stage III cervical cancer treated with chemoradiation three years prior. Her past surgical history included a retropubic porcine dermis Pelvilace sling (Bard Medical, Covington GA) placement and cystoscopy 10 months prior to presentation. The clinical presentation was highly suggestive of local reaction to porcine dermis suburethral sling, however the diagnosis of recurrence of cervical cancer could not be excluded. The patient underwent definitive management with incision and drainage of a large pocket of pus, suprapubic exploration and resection of an indurated mass. The pathology report revealed poorly formed granuloma and no evidence of cancerous cells. The patient had an uncomplicated postoperative course and remains continent and asymptomatic 24 months post operatively.
\end{abstract}

Conclusion: We present a rare case of granuloma formation secondary to reaction to porcine dermis graft that presented as a suprapubic mass.

\author{
Volume 5 Issue 4 - 2016
}

\author{
Lindsay Martin, Maria Andrikopoulou, \\ George Lazarou \\ Department of Obstetrics \& Gynecology,Winthrop University \\ Hospital, USA
}

Correspondence: George Lazarou, Chief of Gynecology, Director of Urogynecology \& Reconstructive Pelvic Surgery, Winthrop-University Hospital, 259 First Street, Mineola, New York, II50I, Tel 5I6-663-2655, Fax 516-742-782I, Email glazarou@winthrop.org

Received: August 23, 2016 | Published: November 07, 2016

\section{Case}

A 43-year-old multipara presented 10 months post-operative from a retropubic sling with complaint of a growing left suprapubic mass. Her medical history was significant for stage III cervical cancer treated with chemoradiation, including vaginal brachytherapy three years prior. The patient was followed by gynecologic oncology and had no recurrent malignancy, but had developed a short vagina postradiation. The patient had undergone a retropubic porcine dermis Pelvilace suburethral sling (Bard Medical, Covington GA) placement and cystoscopy for stress urinary incontinence (SUI). The procedure was complicated by fibrotic vaginal tissue and a recognized 3 millimeter right-sided dome cystotomy, requiring modification to a transobturator approach on the right side. The modified transobturator approach was performed by securing the right arm of the sling to the Obturator Internus fascia with 2-0 polydioxanone sutures using the Capio device. Her post-operative course was uncomplicated until the appearance of a left-sided suprapubic mass 10 months after sling placement.

On examination, the patient was comfortable, afebrile and vital signs were within normal limits. A $3 \mathrm{~cm}$ left suprapubic mass without fluctuation was noted. There was no evidence of local inflammation or abscess. Speculum exam revealed a short vagina due to previous radiation, with no evidence of any foreign body and no evidence of vaginitis. On bimanual examination there were no appreciable vaginal or pelvic masses. A pelvic MRI revealed a nonspecific suprapubic left subcutaneous inflammation extending to the sub-vaginal tissue (Figure 1). The clinical presentation was highly suggestive of local reaction to the porcine dermis arm on the left side. Because of the patient's history of cervical cancer, gynecologic oncology was consulted to rule out recurrence of cervical cancer. The surgical team was also consulted to rule out a hernia.

The patient was initially managed conservatively with nonsteroidals and a one-week course of oral levofloxacin. She underwent an office cystourethroscopy which was unremarkable. Fine needle aspiration of the mass was performed which showed squamous cells without atypia. Failure of resolution of the mass with conservative management required definitive treatment with suprapubic surgical exploration. The patient was taken to the Operating Room, an incision and drainage of a large pocket of pus was performed, and exploration and resection of an indurated mass in the subcutaneous layer was completed. The final pathology report revealed acute and chronically inflamed fibroadipose tissue with poorly formed granuloma (Figure 2). There was no evidence of carcinoma and all wound cultures were negative. Her post-operative course was uncomplicated and the patient was discharged home same day with instructions. The mass did not recur and the patient remains asymptomatic twenty-four months post operatively. Further, the patient remained continent without any urinary symptoms.

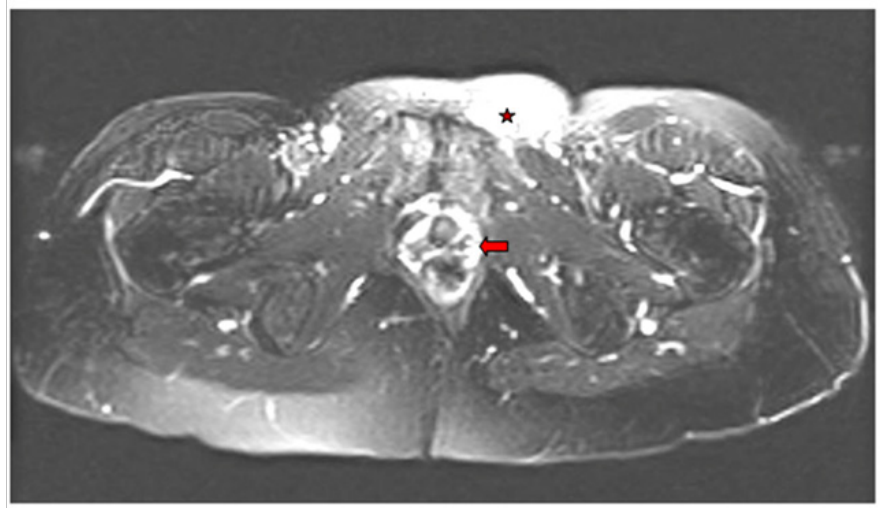

Figure I T2 noncontrast Axial MRI of the pelvis showing nonspecific subcutaneous edema of the left suprapubic area (asterisks) and sub-vaginal tissues (arrow). 


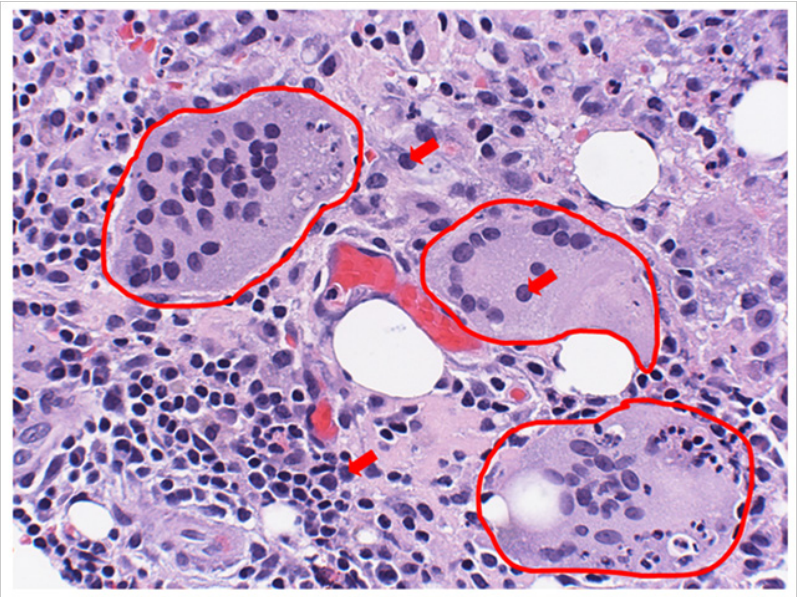

Figure 2 Hematoxylin-eosin stain of the groin granuloma (Magnification $x 400$ ). Note the multinucleated giant cells (outlined), lymphocytes (arrows), neutrophil (arrow head).

\section{Discussion}

Tension free slings are considered the "preferred" approach for the correction of stress urinary incontinence with urethral hypermobility. Unfortunately, among others, the following uncommonly reported postoperative complications of foreign body vaginal extrusion, urethral or intravesical erosion, and voiding dysfunction have been reported. ${ }^{1}$ Additionally, another rare but reported possible complication of tension free slings is fistula formation. ${ }^{2}$ Such reports of vesicovaginal fistulas exist after complicated slings especially when additional risk factors such as multiple previous pelvic surgeries or prior pelvic irradiation exist. ${ }^{3}$ In our patient, the prior history of vaginal radiation excluded the use of synthetic sling and the patient had declined an autologous rectus fascia sling.

Various biologic materials such as autologous, allograft or xenograft materials have been used for suburethral slings with varying results. Biologic slings may be considered the gold standard in patients with previous mesh complications, history of previous radiation, severe vaginal atrophy, immunosuppression, or in patients with increased risk of delayed healing or infection. In autografts, tissue can be used from the patient or someone genetically identical, such as rectus fascia, fascia lata, vaginal tissue, dermis or tendon. ${ }^{4}$ In allografts, a tissue graft can be donated from a donor of the same species as the recipient but not genetically identical, such as a cadaver while a xenograft is obtained from a donor of a different species. For example, porcine collagen in xenografts has a $95 \%$ homology to human collagen, and is easy to handle because it rehydrates quickly. ${ }^{5}$ Autologous slings are reported to be equal in efficacy as synthetic slings in short, medium and longterm. ${ }^{6}$ In general, minimally invasive slings have decreased operating time, lower rates of post-operative bladder dysfunction and detrusor symptoms, as well as fewer perioperative complications, apart from bladder perforation. ${ }^{7}$

Grafts used in pelvic reconstructive surgery should be biologically compatible and cause minimal inflammatory and cellular reaction. While synthetic grafts activate leukocytes and initiate a $\mathrm{T}$ helper type 1 response, biologic grafts induce a T helper type 2 humoral immune response. Previous studies examining histologic changes of graft materials for slings indicate that porcine dermis grafts have a high propensity to encapsulate. In addition they show no fibroblast infiltration, no inflammatory reaction and no foreign body reaction. ${ }^{8}$
Granuloma formation after a midurethral sling is a rare complication. ${ }^{9}$ As described by Woodruff et al., porcine dermis grafts tend to encapsulate, but should not cause inflammatory or foreign body reaction. Further, transvaginal mesh repairs for correction of pelvic organ prolapse exhibit very low rates of granuloma formation. ${ }^{10}$ According to literature, there is limited data available and there is paucity of information to justify routine use of xenografts over synthetic grafts. ${ }^{11}$ Apart from that, latest studies support that only macroporous monofilament slings are responsible for continuous inflammatory reactions. ${ }^{12}$ However data on granuloma formation and biologic slings are lacking.

Tissue specimens from patients who had explanted cross-linked porcine dermis as suburethral slings using Hematoxylin and Eosin staining revealed limited collagen remodeling and variable tissue reactions. ${ }^{13}$ Further, the use of xenograft porcine dermis in our case with the findings of squamous cells on fine needle aspiration may suggest some graft remnant 10 months after the surgery. According to the literature, histologic host response to implanted xenograft depends mainly on chemical cross-linking and porosity and limited to the following four responses: resorption, incorporation, encapsulation and mixed. ${ }^{14}$

It is our opinion that the porcine dermis did not incorporate and due to the patient's previous pelvic radiation with resulting fibrosis and decreased vascularization ultimately led to the granuloma formation in the subcutaneous layer on the left suprapubic area. Our modification of anchoring the sling arm to the obturator internus fascia on the right side avoided granuloma formation on that side.

With the increasing frequency of surgical management of stress incontinence, it is important for surgeons to recognize and treat all postoperative complications associated with suburethral slings. As the long term complications of biologic slings are still unclear, more studies are needed to evaluate the efficacy and safety of the various biologic grafts.

\section{Acknowledgments}

None.

\section{Conflicts of interest}

None.

\section{References}

1. Cetinel B, Tarcan T. Management of Complications after Tension-Free Midurethral Slings. KoreanJ Urol. 2013;54(10):651-659.

2. Reisenauer C, Janowitz J, Wallwiener D, et al. Urethrovaginal fistulae associated with tension-free vaginal tape procedures: a clinical challenge. Int Urgynecol Journal. 2014;25(3):319-322.

3. Perez CA, Grisby PW, Camel HM, et al. Irradiation alone or combined with surgery in stage IB, IIA, and IIB carcinoma of uterine cervix: update of a nonrandomized comparison. Int J Radiat Oncol Biol Phys. 1995;31(4):703-716.

4. Cox A, Herschorn S, Lee L. Surgical management of female SUI: Is there a gold standard? Nature Reviews Urology. 2013;10(2):78-89.

5. Jakus SM, Shapiro A, Hall CD. Biologic and Synthetic Graft Use in Pelvic Surgery: A Review. Obstet Gynecol Surv. 2008;63(4):253-266.

6. Khan ZA, Nambiar A2, Morley R3, et al. Long-term follow-up of a multicentre randomised controlled trial comparing tension-free vaginal tape, xenograft and autologous fascial slings for the treatment of stress urinary incontinence in women. BJU Int. 115(6)968-977. 
7. Rehman H, Bezerra CC, Bruschini $\mathrm{H}$, et al. Traditional suburethral sling operations for urinary incontinence in women. Cochrane Database Syst Rev. 2011;19(1):CD001754.

8. Reisenauer C, Janowitz J, Wallwiener D, et al. Urethrovaginal fistulae associated with tension-free vaginal tape procedures: a clinical challenge. Int Urgynecol Journal. 2014;25(3):319-322.

9. Perez CA, Grisby PW, Camel HM, et al. Irradiation alone or combined with surgery in stage IB, IIA, and IIB carcinoma of uterine cervix: update of a nonrandomized comparison. Int J Radiat Oncol Biol Phys. 1995;31(4):703-716.

10. Woodruff AJ, Cole EE, Dmochowski RR, et al. Histologic Comparison of Pubovaginal Sling Graft Materials: A Comparative Study. J Urol. 2008;72(1):85-89.
11. Muffly M, Barber M. Insertion and removal of vaginal mesh for pelvic organ prolapse. Cl Obstet. 2010;53(1):99-114.

12. Elmer C, Blomgren B, Falconer C, et al. Histological inflammatory response to transvaginal polypropylene mesh for pelvic reconstructive surgery. J Urol. 2009;181(3):1189-1195.

13. Gandhi S, Kubba LM, Abramov Y, et al. Histopathologic changes of porcine dermis xenografts for transvaginal suburethral slings. Am J Obstet Gynecol. 2005;192(5):1643-1648.

14. Haylen BT, de Ridder D, Freeman RM, et al. An International Urogynecological Association (IUGA)/International Continence Society (ICS) joint report on the terminology for pelvic floor dysfunction. Int Urogynecol J. 2010;21(1):5-26. 